EPJ Web of Conferences 60, 12003 (2013)

DOI: $10.1051 /$ epjconf $/ 20136012003$

(C) Owned by the authors, published by EDP Sciences, 2013

\title{
Higgs Effective Lagrangian
}

\author{
Margherita Ghezzi ${ }^{1}$ \\ ${ }^{1}$ Dipartimento di Fisica, Università di Roma La Sapienza and INFN, Roma, Italy
}

\begin{abstract}
We present a short overview of the main aspects of the Higgs Effective Lagrangian. In particular we highlight a strategy to determine whether the electroweak symmetry breaking sector is controlled by a strong or a weak dynamics. We give an estimate of the effects of the effective operators on physical observables and review the most important bounds on the Wilson coefficients of the effective operators.
\end{abstract}

\section{The Effective Lagrangian}

After the discovery of a boson with mass $m_{h} \simeq 125 \mathrm{GeV}$ by both the ATLAS [1] and CMS [2] collaborations, it is of crucial importance to find the best strategy to study the properties of the new particle, and to investigate the nature of the mechanism behind the electroweak (EW) symmetry breaking. As the production cross section and decay rates of the new boson are compatible with those of the Standard Model (SM) Higgs boson, and no hint of the existence of additional new resonances has revealed itself yet, a parametrization in terms of effective operators seems to be the most appropriate approach to investigate the properties of the new boson. Such an effective description is valid as long as New Physics (NP) states appear at a scale $M \gg m_{h}$, and is based on an expansion in the number of fields and derivatives [3]. The detailed form of the effective Lagrangian depends on which assumptions are made. In the light of the current experimental measurements, it is reasonable to assume that the new boson $h$ is a CP-even scalar that forms an $S U(2)_{L}$ doublet together with the longitudinal polarizations of the $W$ and $Z$, so that at high energies the $S U(2)_{L} \times U(1)_{Y}$ EW symmetry is linearly realized. Under these assumptions, the Higgs Effective Lagrangian (HEL) is formed by a sum of operators of increasing dimensionality, where the leading NP effects are given by dimension- 6 operators.

The most general and systematic classification of the dimension-6 operators for a weak doublet $H$ was made in Ref. [4]. Subsequent analysis pointed out the presence of some redundant operators, and a minimal and complete list of operators was finally provided in Ref. [5]. A convenient basis of operators for Higgs physics was discussed in Ref. [6], assuming that the Higgs boson is a CP-even weak doublet and the baryon and lepton numbers are conserved. This basis was recently proposed again in Ref. [7], where a deep analysis of the operators which affect the Higgs physics is provided. In this proceeding we will summarize the main results pointed out in Ref. [7].

\section{Estimates of the Wilson Coefficients}

In order to investigate the nature of the dynamics behind the EW symmetry breaking, it can be crucially helpful to identify which operators of the HEL can probe the strength of the Higgs couplings to the states belonging to the new dynamics, and which operators are sensitive only to the mass scale $M$. This is of key importance to distinguish between weakly-coupled UV completions of the SM, like Supersymmetric (SUSY) theories, and theories where the EW symmetry is broken by a new stronglyinteracting dynamics which forms the Higgs boson as a bound state $[6,8-10]$. These are the two most compelling scenarios put forward to solve the hierarchy problem of the SM.

We start recalling the classification of the dimension6 operators proposed in Ref. [6], that is based on an expansion of the HEL in the number of fields and derivatives: any extra derivative costs a factor $1 / M$, and any extra Higgs field brings a factor $1 / f \equiv g_{*} / M$, where $g_{*} \leq 4 \pi$ is the generic coupling of the Higgs boson to the NP states. Through this simple naive dimensional analysis, the authors of Ref. [6] could write a dimensional pre-factor in front of the effective operators and define the Wilson coefficients to be of $O(1)$. A slightly different approach was followed in Ref. [7], where the pre-factors were defined in terms of the well-known SM parameters $m_{W}$ and $v$ (instead of $M$ and $f$ ) in order to provide a suitable framework for model-independent studies. It follows that in this case the Wilson coefficients $\bar{c}_{i}$ are not of $O(1)$, as they contain all the dependence on the model-dependent parameters $M$ and $f$ (or, to be more precise, the dependence on the ratios $M / m_{W}$ and $\left.f / v\right)$. This parametrization is also appropriate for the implementation of the HEL into automatic tools for the computation of physical quantities, like the Higgs production cross sections and decay rates. As an example, see the program eHDECAY [11], available now at the following URL: http://www.itp.kit.edu/ maggie/eHDECAY/. It is a modified version of the program HDECAY [12, 13], which includes the full list of leading bosonic operators from the 
HEL, as well as all the available QCD and EW higherorder contributions.

Hereafter we will adopt the parametrization suggested in Ref. [7], and in particular we will refer to the Effective Lagrangian Eqs. (2.1)-(2.4) of the Ref [7]. With those definitions and according to the naive power counting one estimates:

$$
\begin{aligned}
& \bar{c}_{H}, \bar{c}_{T}, \bar{c}_{6}, \bar{c}_{\psi} \sim O\left(\frac{v^{2}}{f^{2}}\right), \quad \bar{c}_{W}, \bar{c}_{B} \sim O\left(\frac{m_{W}^{2}}{M^{2}}\right), \\
& \bar{c}_{H W}, \bar{c}_{H B}, \bar{c}_{\gamma}, \bar{c}_{g} \sim O\left(\frac{m_{W}^{2}}{16 \pi^{2} f^{2}}\right), \quad \bar{c}_{H u d} \sim O\left(\frac{\lambda_{u} \lambda_{d}}{g_{*}^{2}} \frac{v^{2}}{f^{2}}\right), \\
& \bar{c}_{H \psi}, \bar{c}_{H \psi}^{\prime} \sim O\left(\frac{\lambda_{\psi}^{2}}{g_{*}^{2}} \frac{v^{2}}{f^{2}}\right), \quad \bar{c}_{\psi W}, \bar{c}_{\psi B}, \bar{c}_{\psi G} \sim O\left(\frac{m_{W}^{2}}{16 \pi^{2} f^{2}}\right),
\end{aligned}
$$

where $\lambda_{\psi}$ denotes the coupling of a generic SM fermion $\psi$ to the new dynamics. These estimates hold at the scale $M$, at which the HEL is matched onto explicit models. At the EW scale they achieve contributions from the renormalization-group flow, which give in general subdominant corrections. Notice that the estimates in (1) can play a role in understanding the nature of the EW symmetry breaking sector, as the dependence on $g_{*}$ is shown explicitly. Indeed, it turns out that if the Higgs boson is a composite state of a new dynamics at the scale $M$, it is natural to expect $g_{*} \gg 1$ (i.e. $f \ll M$ ). In this case, the operators with extra powers of $H$ give the leading corrections to low-energy observables. On the other hand, in weakly-coupled completions of the SM, where $g_{*} \sim g$, all operators with the same dimension can be equally important.

It follows from the estimate (1) that in the case of a strongly-interacting light Higgs boson the leading NP effects in Higgs observables are parametrized by the operators $O_{H, T, 6, \psi}$, and by the fermionic operators $O_{H \psi, H \Psi, H u d}$ and $O_{H \psi, h \Psi}^{\prime}$ if the SM fermions strongly couple to the new dynamics. On the other hand, $\bar{c}_{H W, H B, g, \gamma}$ and the coefficients of the dipole operators are suppressed by an additional loop factor $\left(g_{*}^{2} / 16 \pi^{2}\right)$, as the corresponding operators cannot be generated at tree level in a minimally coupled theory.

A special and phenomenologically motivated case is represented by theories where the Higgs doublet is a composite Nambu-Goldstone boson of a spontaneouslybroken symmetry $\mathcal{G} \rightarrow \mathcal{H}$ of the strong dynamics [6, 810]. For these models the scale $f$ must be identified with the decay constant associated with the spontaneous breaking, and the naive estimate of the Wilson coefficients $\bar{c}_{i}$ is modified by the request of invariance under $\mathcal{G}$ in the limit of vanishing explicit breaking. At the level of dimension6 operators, $O_{\gamma}, O_{g}, O_{6}, O_{u, d, l}$ and the dipole operators violate the shift symmetry $H^{i} \rightarrow H^{i}+\zeta^{i}\left(\zeta^{i}=\right.$ const. $)$ that is included as part of the $\mathcal{G} / \mathcal{H}$ transformations. This means that they cannot be generated in absence of an explicit breaking of the global symmetry. It follows that the naive estimates of the operators $O_{\gamma}$ and $O_{g}$ carry in this case an additional suppression factor [6],

$$
\bar{c}_{\gamma}, \bar{c}_{g} \sim O\left(\frac{m_{W}^{2}}{16 \pi^{2} f^{2}}\right) \times \frac{g_{G}^{2}}{g_{*}^{2}},
$$

where $g_{G}$ denotes any weak coupling that breaks the Goldstone symmetry (one of the SM weak couplings in minimal models, i.e. the SM gauge couplings or the Yukawa couplings). On the other hand, the operators $O_{6}, O_{\psi}, O_{\psi G}$, $O_{\psi W}, O_{\psi B}$ have been defined so that their pre-factor already includes one spurion coupling, precisely the Higgs quartic coupling $\lambda$ in $O_{6}$, and the Yukawa coupling $y_{\psi}$ in the other operators - indeed, both these couplings vanish for an exact Nambu-Goldstone boson. The estimates of the corresponding coefficients $\bar{c}_{6}, \bar{c}_{\psi}, \bar{c}_{\psi G}, \bar{c}_{\psi W}, \bar{c}_{\psi B}$ given in Eq. (1) are thus not modified.

\section{Bounds on flavor-preserving operators}

We report here the most interesting constraints on the Wilson coefficients $\bar{c}_{i}$ obtained from current experimental results. The whole set of bounds on flavor-conserving operators is presented in Ref. [7], while for a discussion on the bounds on flavor-violating operators see for example Refs. $[14,15]$. Among the strongest bounds are those on operators that modify the vector-boson self-energies. The operator $O_{T}$, for example, violates the custodial symmetry [16] and contributes to the EW parameter $\epsilon_{1}[17,18]$. From the EW fit performed in Ref. [19], it follows, with $95 \%$ probability,

$$
\Delta \epsilon_{1} \equiv \Delta \rho=\bar{c}_{T}\left(m_{Z}\right), \quad-1.5 \times 10^{-3}<\bar{c}_{T}\left(m_{Z}\right)<2.2 \times 10^{-3} .
$$

Such a stringent bound can be more naturally satisfied by assuming that the dynamics at the scale $M$ possesses an (at least approximate) $S U(2)_{V}$ custodial invariance. In this case $c_{T}(M)=0$, and a non-vanishing value will be generated through the renormalization-group flow of this Wilson coefficient down to $m_{Z}$ in the presence of an explicit breaking of the custodial symmetry. The EW precision tests also imply a strong bound on $O_{W}+O_{B}$ [6], since this linear combination contributes to the parameter $\epsilon_{3}[17,18]$. With $95 \%$ probability, one has [19]:

$$
\begin{gathered}
\Delta \epsilon_{3}=\bar{c}_{W}\left(m_{Z}\right)+\bar{c}_{B}\left(m_{Z}\right), \\
-1.4 \times 10^{-3}<\bar{c}_{W}\left(m_{Z}\right)+\bar{c}_{B}\left(m_{Z}\right)<1.9 \times 10^{-3} .
\end{gathered}
$$

From the tree-level estimate of $\bar{c}_{W, B}$ reported in Eq. (1), and assuming an approximate custodial invariance to suppress $\bar{c}_{T}$ as explained above, it follows that Eqs. (3) and (4) set a lower bound $M \gtrsim$ a few $\mathrm{TeV}$. This bound is quite robust and can be avoided only in weakly-coupled UV completions where an extra symmetry protection suppresses the leading contribution to $\bar{c}_{W, B}$ by an additional loop factor. Notable examples are SUSY theories with $R$-parity.

For the fermionic operators strong constraints come from the Z-pole measurements, as this class of operators modify the couplings of the $\mathrm{Z}$ boson to quarks and leptons:

$$
\frac{\delta g_{L \psi}}{g_{L \psi}}=\frac{1}{2} \frac{\left(-\bar{c}_{H \Psi}+2 T_{3 L} \bar{c}_{H \Psi}^{\prime}\right)}{T_{3 L}-Q \sin ^{2} \theta_{W}}, \quad \frac{\delta g_{R \psi}}{g_{R \psi}}=\frac{1}{2} \frac{\bar{c}_{H \psi}}{Q \sin ^{2} \theta_{W}},
$$


where $T_{3 L}$ and $Q$ are respectively the $S U(2)_{L}$ and electric charges of the fermion $\psi$, and $\Psi=\{L, q\}$ is the $S U(2)_{L}$ doublet to which $\psi_{L}$ belongs. The bounds, obtained performing a fit on the coefficients $\bar{c}_{H \psi}, \bar{c}_{H \Psi}$ and $\bar{c}_{H \Psi}^{\prime}$, are shown in Ref. [7]. Among them, the weakest is the one on the operator $O_{H b}$, which modifies the coupling of $b_{R}$ to the $Z$ boson:

$$
-0.07<\bar{c}_{H b}<-0.005 \text {. }
$$

According to the estimates (1), such a set of bounds puts a strong limit on the couplings of the SM fermions to the new dynamics. Unless the scale of NP is very large, or some specific symmetry protection is at work at the UV scale, it follows that the SM fermions must be very weakly coupled to the new dynamics $\left(\lambda_{\psi} \ll g_{*}\right)$, with the possible exception of the top quark, as the operator $O_{H t}$ is totally unconstrained by EW data (but also substantially irrelevant for the Higgs physics).

Finally, the constraints on the dipole operators come mainly from the experimental limits on electric dipole moments and anomalous magnetic moments. It turns out that the bounds involving the light quarks $u$ and $d$ are very strong, implying, according to the naive estimate (1), a bound on $(v / f)^{2}$ at the level of $10^{-3}$. In natural extensions of the SM, such a strong limit clearly points to a symmetry protection mechanism. Among the heavier quarks, the most interesting bounds are those involving the top quark. These come from the neutron electric dipole moment,

$$
-1.39 \times 10^{-4}<\operatorname{Im}\left(\bar{c}_{t G}\right)<1.21 \times 10^{-4},
$$

the $b \rightarrow s \gamma$ and $b \rightarrow s l^{+} l^{-}$rates,

$$
-0.057<\operatorname{Re}\left(\bar{c}_{t W}+\bar{c}_{t B}\right)-2.65 \operatorname{Im}\left(\bar{c}_{t W}+\bar{c}_{t B}\right)<0.20
$$

and the $t \bar{t}$ cross sections measured at Tevatron and LHC,

$$
-6.12 \times 10^{-3}<\operatorname{Re}\left(\bar{c}_{t G}\right)<1.94 \times 10^{-3} \text {. }
$$

All these bounds are computed at $95 \%$ probability and at the low-energy scale. It is interesting to notice that the bounds on $\bar{c}_{t G}, \bar{c}_{t W}$ and $\bar{c}_{t B}$ are about one order of magnitude weaker than the size expected from the naive estimate (1) with $(v / f)^{2} \sim 0.1$.

\section{Effects on physics observables}

In this section we will consider the contribution of the effective operators to physical observables, with particular attention to the Higgs decay rates. We start considering the operators $O_{H}, O_{T}, O_{u, d, l}$ and $O_{6}$, which modify the tree-level couplings of the Higgs boson to fermions, vector bosons and itself. In the unitary gauge and upon canonical normalization of the Higgs kinetic term, the Lagrangian reads [20]

$$
\begin{aligned}
\mathcal{L}= & \frac{1}{2} \partial_{\mu} h \partial^{\mu} h-\frac{1}{2} m_{h}^{2} h^{2}-c_{3} \frac{1}{6}\left(\frac{3 m_{h}^{2}}{v}\right) h^{3}+\ldots \\
& +m_{W}^{2} W_{\mu}^{+} W^{-\mu}\left(1+2 c_{W} \frac{h}{v}+\ldots\right) \\
& +\frac{1}{2} m_{Z}^{2} Z_{\mu} Z^{\mu}\left(1+2 c_{Z} \frac{h}{v}+\ldots\right) \\
& -\sum_{\psi=u, d, l} m_{\psi^{(i)}} \bar{\psi}^{(i)} \psi^{(i)}\left(1+c_{\psi} \frac{h}{v}+\ldots\right)+\ldots
\end{aligned}
$$

where the Higgs couplings $c_{i=W, Z, \psi, 3}$, have been defined such that $c_{i}=1$ in the SM. The shifts from the SM value are of order

$$
\delta c_{i} \sim \frac{g_{*}^{2} v^{2}}{M^{2}}=\frac{v^{2}}{f^{2}} .
$$

Hence, measuring the Higgs couplings probes the strength of its interactions to the new dynamics.

The operators $O_{W}$ and $O_{B}$ can be generated at treelevel by the exchange of heavy particles, like, for example, spin-1 states. In the unitary gauge they can be written in terms of the following three operators:

$$
\left(D^{\mu} W_{\mu \nu}^{+}\right) W^{-v} h, \quad\left(\partial^{\mu} Z_{\mu v}\right) Z^{v} h, \quad\left(\partial^{\mu} \gamma_{\mu \nu}\right) Z^{v} h
$$

plus terms with zero or two Higgs fields. It can be easily seen that these operators give corrections to the tree-level Higgs couplings and generate quartic interactions of the type $h V \bar{\psi} \psi$, that contribute to the three-body decays $h \rightarrow$ $V V^{*} \rightarrow V \psi \bar{\psi}$. Indeed, it turns out that $O_{W}$ and $O_{B}$ can be expressed in terms of other operators of the HEL:

$$
\begin{gathered}
O_{W}=-6 O_{H}+2\left(\left(O_{u}+O_{d}+O_{l}\right)+\text { h.c. }\right) \\
-8 O_{6}+O_{H q}^{\prime}+O_{H L}^{\prime}, \\
O_{B}=2 \tan ^{2} \theta_{W}\left(-O_{T}+O_{H \Psi}^{Y}\right),
\end{gathered}
$$

where $O_{H \psi}^{Y} \equiv \sum_{\psi} Y_{\psi} O_{H \psi}$. The contribution of $O_{W, B}$ to the Higgs decay rates is of order $m_{W}^{2} / M^{2}$, i.e. these operators are sensitive only to the value of the NP scale M:

$$
\left.\frac{\delta \Gamma(h \rightarrow V V)}{\Gamma(h \rightarrow V V)}\right|_{O_{W}, O_{B}} \sim O\left(\frac{m_{W}^{2}}{M^{2}}\right),
$$

where in this case $V V=W^{(*)} W^{*}, Z^{(*)} Z^{*}, Z^{(*)} \gamma, \gamma \gamma$.

Now let us move to the operators $O_{H W}, O_{H B}, O_{\gamma}$ and $O_{g}$. They are generated at the one-loop level, and in the unitary gauge are rewritten in terms of

$$
W_{\mu \nu}^{+} W^{-\mu v} h, \quad Z_{\mu \nu} Z^{\mu v} h, \quad \gamma_{\mu \nu} \gamma^{\mu v} h, \quad Z_{\mu \nu} \gamma^{\mu v} h
$$

plus other terms with zero or two Higgs fields. As implied from the naive estimates (1), the contribution of $O_{H W, H B, \gamma}$ to the $W W$ and $Z Z$ inclusive rates is of order $(V V=W W, Z Z)$

$$
\left.\frac{\delta \Gamma(h \rightarrow V V)}{\Gamma(h \rightarrow V V)}\right|_{O_{\gamma}, O_{H W}, O_{H B}} \sim O\left(\frac{m_{W}^{2}}{16 \pi^{2} f^{2}}\right) .
$$


This effect is sensitive to the interaction strength $g_{*}$, but also suppressed by the loop factor. On the other hand, the processes $h \rightarrow \gamma \gamma, h \rightarrow Z \gamma$ and $h \rightarrow g g$ can in principle test the Higgs interaction strength more powerfully, since they arise at the one-loop level in the SM. Naively one gets:

$$
\left.\frac{\delta \Gamma(h \rightarrow g g, \gamma \gamma, Z \gamma)}{\Gamma(h \rightarrow g g, \gamma \gamma, Z \gamma)}\right|_{O_{g}, O_{\gamma}, O_{H W}, O_{H B}} \sim O\left(\frac{v^{2}}{f^{2}}\right),
$$

but if the Higgs boson is a Nambu-Goldston boson, the coefficients $\bar{c}_{g}$ and $\bar{c}_{\gamma}$ are further suppressed by a factor $\left(g_{\mathscr{G}} / g_{*}\right)^{2}$, according to the estimate (2). This implies that in this class of theories the corrections to $\Gamma(h \rightarrow \gamma \gamma)$ and $\Gamma(h \rightarrow g g)$ depend only on the scale $M$, and we are left only with $\Gamma(h \rightarrow Z \gamma)$ to test the value of $g_{*}$.

On the fermionc-operator side, we see that the operators of the form $\left(\bar{\psi} \gamma^{\mu} \psi\right)\left(H^{\dagger} \overleftrightarrow{D^{\mu}} H\right)$ are sensitive to the strength of the couplings of the Higgs boson and of the SM fermions to the new dynamics. They lead to contact corrections to the three-body decays $h \rightarrow V V^{*} \rightarrow V \psi \psi$ which are naively of order

$$
\frac{\delta \Gamma(h \rightarrow V \bar{\psi} \psi)}{\Gamma(h \rightarrow V \bar{\psi} \psi)} \sim O\left(\frac{v^{2}}{f^{2}} \frac{\lambda_{\psi}^{2}}{g_{*}^{2}}\right) .
$$

Compared to the corrections from $O_{W}$ and $O_{B}$, the effect of the fermionic operators is potentially enhanced by a factor $\left(\lambda_{\psi}^{2} / g^{2}\right)$. In practice, the possibility of large fermionic couplings $\lambda_{\psi}$ is strongly constrained by LEP, as mentioned above. Hence, the only way to have a large degree of compositeness of either the left- or the right-handed quarks is to have some protective symmetry which prevents the generation of this kind of operators.

Finally, let us consider the dipole operators. While those involving light fermions are strongly constrained by experimental data, potentially sizable effects could come from operators with the top quark. For example, the estimates of the contributions of $O_{t G}$ to $g g \rightarrow h, g g \rightarrow t \bar{t}$ and $g g \rightarrow t \bar{t} h$ are

$$
\begin{gathered}
\frac{\delta \sigma(g g \rightarrow h)}{\sigma(g g \rightarrow h)} \sim \hat{c}_{t G}, \quad \frac{\delta \sigma(g g \rightarrow t \bar{t})}{\sigma(g g \rightarrow t \bar{t})} \sim \hat{c}_{t G} \frac{\sqrt{s}}{m_{t}} \\
\frac{\delta \sigma(g g \rightarrow t \bar{t} h)}{\sigma(g g \rightarrow t \bar{t} h)} \sim \hat{c}_{t G} \frac{s}{m_{t}^{2}}
\end{gathered}
$$

with $\hat{c}_{t G} \equiv \operatorname{Re}\left(\bar{c}_{t G}\right)\left(m_{t}^{2} / m_{W}^{2}\right) \sim m_{t}^{2} /\left(16 \pi^{2} f^{2}\right) \simeq 3 \times$ $10^{-3}\left(v^{2} / f^{2}\right)$. One immediately notice that the study of the differential distributions could be a successful strategy. The NP contribution to the process $g g \rightarrow t \bar{t} h$ can in principle get the largest enhancement from a cut on $\sqrt{s}$, but the small rate might limit the sensitivity achievable at the LHC. Thus, the most sensitive process is perhaps $g g \rightarrow t \bar{t}$, in particular at large invariant mass, although the current experimental precision is still insufficient to constrain $(v / f)$.

\section{The HEL beyond the tree level}

In conclusion of this short overview of the main aspects of the HEL, we recall a few issues, treated more exhaustively in Ref. [7], about the HEL beyond the tree level, as required to make Higgs precision physics without assuming the validity of the SM.

The first difficulty that one has to face is the presence of multiple expansion parameters. In fact, the validity of the HEL is based on the perturbative expansion in the SM couplings, $\alpha_{S M} /(4 \pi)$, in powers of $E / M$ (derivative expansion) and in powers of $v / f$, under the condition $v \ll f$. All these expansion parameters must be properly taken into account when performing calculations. Furthermore, the non-renormalizability of the effective theory implies the presence of additional divergences compared to the SM case which must be absorbed by a renormalization of the Wilson coefficients of local operators.

While referring the reader to Ref. [7] for a proper discussion of the renormalization issue, here we will focus on the long-distance contributions. In general, the decay amplitude can be expanded as follows: ${ }^{1}$

$$
A=A_{0}^{S M}+A_{1}^{S M}+\Delta A_{0}+\Delta A_{1}+\ldots
$$

where $A_{0}^{S M}\left(A_{1}^{S M}\right)$ is the tree-level (one-loop) SM amplitude, and $\Delta A_{0}\left(\Delta A_{1}\right)$ is the tree-level (one-loop) contribution from the dimension- 6 operators of the HEL. The dots denote higher-loop contributions as well as the corrections due to higher-order operators.

To be more concrete, let us focus on the decay $h \rightarrow$ $W^{(*)} W^{*}$, keeping in mind that the other processes must be treated in the same spirit. In our example, the operators that can contribute at the tree level are $O_{H}, O_{W}, O_{H W}, O_{\psi W}$, $O_{H \psi}^{\prime}$, as well as $O_{H u d}$ when the off-shell $W$ decays into a pair of quarks. Based on the naive estimates (1), we can quantify the various effects encoded by $\Delta A_{0}$ as follows:

$$
\begin{aligned}
\frac{\Delta A_{0}}{A_{0}^{S M}}\left(W^{(*)} W^{*}\right) & =\hat{c}_{H} \times O\left(\frac{v^{2}}{f^{2}}\right)+\hat{c}_{W} \times O\left(\frac{E^{2}}{M^{2}}\right) \\
& +\hat{c}_{H W} \times O\left(\frac{E^{2}}{16 \pi^{2} f^{2}}\right)+\hat{c}_{H u d} \times O\left(\frac{v^{2}}{f^{2}} \frac{\lambda_{u} \lambda_{d}}{g_{*}^{2}}\right) \\
& +\hat{c}_{H \psi}^{\prime} \times O\left(\frac{v^{2}}{f^{2}} \frac{\lambda_{\psi}^{2}}{g_{*}^{2}}\right)+\hat{c}_{\psi W} \times O\left(\frac{E m_{\psi}}{16 \pi^{2} f^{2}}\right) .
\end{aligned}
$$

Here $E=m_{h}$ is the relevant energy of the process and we have conveniently defined each of the $O(1)$ parameters $\hat{c}_{i}$ to be equal to $\bar{c}_{i}\left(m_{h}\right)$ divided by its naive estimate in Eq. (1). When the Higgs boson is a pseudo NambuGoldstone boson, the two parameters $\hat{c}_{g}$ and $\hat{c}_{\gamma}$ are not of $O(1)$, but are further suppressed by a factor $g_{G}^{2} / g_{*}^{2}$. From Eq. (22) one can see that the contribution of the dipole operators $O_{\psi W}$ is suppressed by $\left(m_{\psi} / m_{h}\right)$ compared to that of $O_{H W}$, while that of $O_{H u d}$ and $O_{H \psi}^{\prime}$ is expected to be small given the existing constraints on the couplings $\lambda_{\psi}$ (see the

\footnotetext{
${ }^{1}$ In the strict sense this equation is valid for the genuine EW corrections only, while for simplicity we include the (IR-divergent) virtual QED corrections to the SM amplitude in the same way. The corresponding real photon radiation contributions to the decay rates are treated in terms of a linear novel contribution to the Higgs coupling for the squared amplitude in order to obtain an infrared finite result. Pure QED corrections factorize as QCD corrections in general so that their amplitudes scale with the modified Higgs couplings. However, they cannot be separated from the genuine EW corrections in a simple way.
} 
discussion in Section 3). Thus, the dominant NP contribution comes from the first three terms of Eq. (22), among which the one proportional to $\bar{c}_{H}$ is the leading effect for $g_{*}>g$. On the other hand, the 1-loop EW amplitude $A_{1}^{S M}$ gives a contribution of order $A_{1}^{S M} / A_{0}^{S M} \sim\left(\alpha_{2} / 4 \pi\right)$. Hence, we see explicitly that $\Delta A_{0}$ and $A_{1}^{S M}$ encode the NLO corrections in the three expansion parameters we are considering: $\alpha_{2} / 4 \pi$ (EW expansion), $E^{2} / M^{2}$ (derivative expansion) and $v^{2} / f^{2}$. The contribution due to 1-loop diagrams with one insertion of the effective vertices has not been computed yet, but we can easily estimate its size:

$$
\begin{aligned}
\frac{\Delta A_{1}}{A_{0}^{S M}\left(W^{(*)} W^{*}\right)=} & \hat{c}_{H} \times O\left(\frac{v^{2}}{f^{2}} \frac{\alpha_{2}}{4 \pi}\right)+\hat{c}_{u} \times O\left(\frac{v^{2}}{f^{2}} \frac{\alpha_{2}}{4 \pi}\right) \\
& +\hat{c}_{6} \times O\left(\frac{v^{2}}{f^{2}} \frac{\alpha_{2}}{4 \pi}\right)+\ldots
\end{aligned}
$$

The terms shown in Eq. (23) arise from the same 1-loop diagrams that give the SM amplitude $A_{1}^{S M}$, where each of the Higgs couplings gets shifted by $\bar{c}_{H}, \bar{c}_{u}$ and $\bar{c}_{6}$. By neglecting the unknown $\Delta A_{1}$ one is omitting terms of order $\left(v^{2} / f^{2}\right)\left(\alpha_{2} / 4 \pi\right)$, that is, of the same size of the tree-level contribution due to the operator $O_{H W}$, see Eq. (22), since $E=m_{h} \approx m_{W}$. This latter contribution can be easily computed and it is included in the formula of the decay rate to $W W$ (and similarly that of $O_{H W}$ and $O_{H B}$ to $Z Z$ is also included) implemented in the program eHDECAY discussed in Ref. [11] and should be seen as the first step towards a full inclusion of the $O\left[\left(v^{2} / f^{2}\right)\left(\alpha_{2} / 4 \pi\right)\right]$ corrections.

Finally, the NLO expression for the decay rate of a Higgs boson to a $W$ pair is given by the following formula:

$$
\begin{aligned}
\Gamma\left(W^{(*)} W^{*}\right) & =\Gamma_{0}^{S M}\left(W^{(*)} W^{*}\right) \\
\times & \left\{1+\frac{2}{\left|A_{0}^{S M}\right|^{2}} \operatorname{Re}\left[\left(A_{0}^{S M}\right)^{*}\left(A_{1}^{S M}+\Delta A_{0}\right)\right]\right. \\
& \left.+O\left(\left(\frac{v^{2}}{f^{2}}\right)^{2},\left(\frac{\alpha_{2}}{4 \pi} \frac{v^{2}}{f^{2}}\right),\left(\frac{\alpha_{2}}{4 \pi}\right)^{2}\right)\right\},
\end{aligned}
$$

where $\Gamma_{0}^{S M}\left(W^{(*)} W^{*}\right)$ denotes the tree-level SM decay rate. For simplicity, we have not shown terms involving powers of $E^{2} / M^{2}$ among the neglected contributions, since for $E=m_{h} \approx m_{W}$ one has $E^{2} / M^{2} \lesssim v^{2} / f^{2}$ if $g_{*} \gtrsim g$. The inclusion of the $O\left(\mathrm{~m}_{h}^{2} / M^{2}\right)$ tree-level correction due to $O_{W}$ is justified as long as $g_{*}<4 \pi$, since it is parametrically larger than the neglected $O\left[\left(v^{2} / f^{2}\right)\left(\alpha_{2} / 4 \pi\right)\right]$ terms by a factor $\left(16 \pi^{2} / g_{*}^{2}\right)$. Notice that in the limit of large deviations of the Higgs couplings from their SM values, $(v / f)^{2} \sim O(1)$, the neglected terms of $O\left[\left(v^{2} / f^{2}\right)\left(\alpha_{2} / 4 \pi\right)\right]$ become as important as those included through $A_{1}^{S M}$. In other words, a proper inclusion of the EW corrections in the limit $v \sim f$ requires a complete 1-loop calculation where each of the diagrams is rescaled by the appropriate coupling factor.

\section{References}

[1] G. Aad et al. [ATLAS Collaboration], Phys. Lett. B 7161 (2012) [arXiv:1207.7214 [hep-ex]].

[2] S. Chatrchyan et al. [CMS Collaboration], Phys. Lett. B 71630 (2012) [arXiv:1207.7235 [hep-ex]].

[3] S. Weinberg, The quantum theory of fields (Univ. Pr., Cambridge, UK, 1996) 489

[4] W. Buchmuller and D. Wyler, Nucl. Phys. B 268621 (1986).

[5] B. Grzadkowski, M. Iskrzynski, M. Misiak and J. Rosiek, JHEP 1010085 (2010) [arXiv:1008.4884 [hep-ph]].

[6] G. F. Giudice, C. Grojean, A. Pomarol and R. Rattazzi, JHEP 0706045 (2007) [hep-ph/0703164].

[7] R. Contino, M. Ghezzi, C. Grojean, M. Muhlleitner and M. Spira, JHEP in press [arXiv:1303.3876 [hep$\mathrm{ph}]]$.

[8] D. B. Kaplan and H. Georgi, Phys. Lett. B 136183 (1984).

[9] R. Contino, Y. Nomura and A. Pomarol, Nucl. Phys. B 671148 (2003) [hep-ph/0306259].

[10] K. Agashe, R. Contino and A. Pomarol, Nucl. Phys. B 719165 (2005) [hep-ph/0412089].

[11] R. Contino, M. Ghezzi, C. Grojean, M. Muhlleitner and M. Spira, in preparation.

[12] A. Djouadi, J. Kalinowski and M. Spira, Comput. Phys. Commun. 10856 (1998) [hep-ph/9704448].

[13] A. Djouadi, M. M. Muhlleitner and M. Spira, Acta Phys. Polon. B 38635 (2007) [hep-ph/0609292].

[14] G. Isidori, Y. Nir and G. Perez, Ann. Rev. Nucl. Part. Sci. 60355 (2010) [arXiv:1002.0900 [hep-ph]].

[15] G. Isidori, arXiv:1302.0661 [hep-ph].

[16] P. Sikivie, L. Susskind, M. B. Voloshin and V. I. Zakharov, Nucl. Phys. B 173189 (1980).

[17] G. Altarelli and R. Barbieri, Phys. Lett. B 253, 161 (1991).

[18] G. Altarelli, R. Barbieri and S. Jadach, Nucl. Phys. B 369, 3 (1992) [Erratum-ibid. B 376, 444 (1992)].

[19] M. Baak, M. Goebel, J. Haller, A. Hoecker, D. Kennedy, R. Kogler, K. Moenig and M. Schott et al., Eur. Phys. J. C 722205 (2012) [arXiv:1209.2716 [hep-ph]].

[20] R. Contino, C. Grojean, M. Moretti, F. Piccinini and R. Rattazzi, JHEP 1005089 (2010) [arXiv:1002.1011 [hep-ph]]. 